\title{
Formación en valores y competencias socioemocionales para docentes tras una década de innovación
}

\author{
Raquel Palomera \\ Doctora en Psicología \\ Universidad de Cantabria - España \\ palomerr@unican.es \\ https://orcid.org/o0oo-0002-6009-6927
}

\section{Elena Briones}

Doctora en Psicología

Universidad de Cantabria - España brionese@unican.es

http://orcid.org/oooo-0003-4521-4582

\section{Alicia Gómez-Linares}

Doctora en Filosofía y Ciencias de la Educación- Pedagogía

Universidad de Cantabria y Danzerti

- España

agomez@dantzerti.eus

https://orcid.org/oooo-0001-6412-4019

Artículo de Revisión

Recepción: 01 de abril de 2019

Aprobación: 03 de julio de 2019

https://doi.org/10.19053/22160159.v10.n25.2019.9116

\section{Resumen}

Un docente necesita: destrezas para comunicarse y relacionarse positivamente con los diversos agentes de la comunidad educativa y alumnado; capacidad para llevar a cabo procesos de autorregulación y héteroregulación; competencia para liderary manejar el aula, además de saber trabajar en equipo; y capacidad de toma de decisiones responsable y ética para la resolución de problemas en el contexto educativo. Desde la nueva materia de Formación en Valores y Competencias Personales para Docentes, se pretende mejorar tanto la calidad de la enseñanza por parte del docente, como el aprendizaje del estudiante. Este último se sitúa en el centro de un proceso de enseñanza-aprendizaje activo, dialógico y colaborativo en su formación en valores y competencias personales, de utilidad tanto para su vida personal como profesional. Este

Referencia bibliográfica para citar este artículo

Palomera, R., Briones, E., \& Gómez-Linares, A. (2019). Formación en valores y competencias socioemocionales para docentes tras una década de innovación. Praxis \& Saber, 10(24), 93-117. https://doi. org/10.19053/22160159.v10.n25.2019.9116 
proyecto ha sido pionero a nivel nacional e internacional por implementar la formación en valores y competencias personales de forma troncal en los estudios de grado en magisterio. En este trabajo presentamos una revisión de las innovaciones que se han generado desde su creación en 2010.

Palabras clave: educación superior, competencias sociales y emocionales, valores, educación emocional, innovación docente

\title{
Values and social-emotional competencies training for teachers after a decade of innovation
}

\begin{abstract}
A teacher needs to have the skills to communicate and to interact positively with different actors in the education community and with students; he needs to have the ability to carry out autoregulation and hetero-regulation processes; he needs to have the competencies to lead and manage the class, he must also know how to work in teams; he needs to have the capacity to make responsible and ethical decisions in order to solve problems in the context of education. The new subject called Values and Personal Competencies Training for Teachers aims both to improve the quality of education on the part of teachers and to improve student learning. The latter is at the heart of an active, dialogical, and collaborative teaching-learning process in their training in values and personal competencies, which are useful both in terms of personal life and in terms of professional life. This is a pioneering project at national and international levels in the implementation of values and personal competencies training on a mandatory basis in teaching degrees. This work presents a review of the resulting innovations since its creation in 2010.
\end{abstract}

Keywords: higher education, social and emotional competencies, values, emotional education, educational innovation 


\section{La formation aux valeurs et aux compétences socio-émotionnelles pour les enseignants après une décennie d'innovation}

\section{Résumé}

Un enseignant a besoin de compétences pour se communiquer et établir une relation positive avec les différents acteurs de la communauté éducative et les apprenants ; il a besoin de capacités pour mettre en œuvre des processus d'autorégulation et d'hétérorégulation; il a besoin de compétences pour diriger et gérer sa classe, il doit en outre savoir comment travailler en équipe ; et il a besoin de la capacité de prises de décisions responsables et éthiques afin de résoudre des problèmes dans le contexte éducatif. La nouvelle matière de Formation aux Valeurs et aux Compétences Personnelles pour les Enseignants vise à améliorer la qualité de l'enseignement de la part de l'enseignant et à améliorer l'apprentissage de l'étudiant. Ce dernier se retrouve au cœur d'un processus d'enseignement-apprentissage actif, dialogique, et collaboratif dans sa formation des valeurs et des compétences personnelles utiles tant pour sa vie personnelle que pour sa vie professionnelle. Ce projet a joué un rôle pionnier au niveau national et international dans la mise en œuvre de la formation aux valeurs et aux compétences personnelles sur une base obligatoire dans les programmes de formation des enseignants. Ce travail présente une révision des innovations développées depuis sa création en 2010.

Mots-clés : éducation supérieur, compétences sociales et émotionnelles, valeurs, éducation émotionnelle, innovation éducative

\section{Formação em valores e competências sócio- emocionais para docentes depois de uma década de inovação}

\section{Resumo}

Um docente precisa: destrezas para comunicar-se e relacionarse positivamente com os diversos agentes da comunidade educativa 
e alunado; capacidade para levar a cabo processos de autoregulação e hétero-regulação; competência para liderar e manejar a sala, além de saber trabalhar em equipe; e capacidade de toma responsável e ética de decisões para a resolução de problemas no contexto educativo. Desde a nova matéria de Formação em Valores e Competências Pessoais para Docentes pretende-se a melhora da qualidade do ensino por parte do docente e a melhora da aprendizagem do estudante. Situa-se a este último no centro de um processo de ensino-aprendizagem ativo, dialógico e colaborativo em sua formação em valores e competências pessoais, de utilidade tanto para sua vida pessoal como profissional. Este projeto tem sido pioneiro a nível nacional e internacional por implementar a formação em valores e competências pessoais de forma principal nos estudos de grau em magistério. Neste trabalho apresentamos uma revisão das inovações que têm gerado desde sua criação em 2010.

Palavras-chave: educação superior, competências sociais e emocionais, valores, educação emocional, inovação docente 


\section{Inclusión de la nueva asignatura en el currículo de formación inicial de docentes}

La Universidad de Cantabria [UC], en consonancia con el Espacio Europeo de Educación Superior [EEES], y consciente de la importancia que tienen determinadas habilidades y competencias transversales para la formación integral de sus titulados y su inserción laboral, decidió diseñar un plan de actuación para la introducción de valores, competencias y destrezas personales específicas en la formación de sus estudiantes, denominado Formación Transversal. Aspectos como la comunicación personal eficaz, la búsqueda de información y creatividad para la solución de problemas, la capacidad de reacción ante situaciones novedosas, y determinadas competencias como el trabajo en equipo, gestión del tiempo, responsabilidad y autogestión, son elementos distintivos de los graduados de la UC. Además, todos los estudiantes tienen la oportunidad de recibir enseñanzas relacionadas con deontología y ética profesional, uso legítimo e ilegítimo de la información, derechos humanos, oportunidades y accesibilidad universal de las personas con diversidad funcional, solidaridad y los valores propios de una cultura de la paz, valores democráticos de cooperación o desarrollo global sostenible. De esta forma se renovaba el sistema de educación al incorporar aspectos como las actitudes, los valores y las habilidades, que permiten a una persona desenvolverse éticamente con eficacia y autonomía en los nuevos entornos, heterogéneos y cambiantes.

En este contexto surge una nueva asignatura denominada Formación en Valores y Competencias Personales para Docentes [FVCPD], que se ha desarrollado desde 2010 con el inicio del Plan Bolonia, en las titulaciones de Grado de Magisterio en Educación Infantil y Grado de Magisterio en Educación Primaria. Se inserta como una materia troncal de 6 ECTS dentro del módulo de Formación Básica del Plan de Estudios de Grados en Magisterio. Hasta la actualidad, 2700 estudiantes y más de una veintena de personal docente e investigador de la UC y de otras universidades, estatales e internacionales, han participado directa o indirectamente en el proyecto.

La materia FVCPD se imparte en el primer semestre del primer curso, con el objeto de desarrollar las competencias personales de los estudiantes y promover un clima positivo desde el inicio 
en el aula. Este clima y competencias adquiridas ayudarán a los estudiantes a gestionar sus propias emociones y afrontar con más éxito las tareas interpersonales y comunicativas propias de las metodologías del EEES - exposiciones, trabajo en equipos, entre otros-, lo que redundará en su propio beneficio académico y personal, así como en un adecuado desarrollo del resto de materias del plan de estudios.

Hasta la fecha, la Facultad de Educación de la UC, es la única de España que ha incorporado en su currículo oficial como materia básica obligatoria el aprendizaje socioemocional de los futuros docentes, dirigido a su propia formación de competencias personales. Las facultades de educación de la Universidad Complutense de Madrid -Entrenamiento en habilidades socialesy La Laguna -Educación Emocional- lo han hecho en forma de optativa. En la Universidad Internacional de Cataluña - La educación emocional en el docente-incorporan la formación como optativa de mención y de forma específica dirigida al desarrollo de competencias emocionales y sociales del propio docente. La Universidad de Huelva propone una asignatura obligatoria - La educación en valores y la práctica de actividad física en colectivos en situación de desigualdad-, dirigida a la educación en valores en estudiantes con necesidades específicas de apoyo educativo.

Además, como explicaremos en el último apartado, nuestro proyecto innovador también se ha aplicado en posgrado en la UC y se ha transferido a otros ámbitos sociales, más allá del contexto universitario.

\section{Necesidad de la formación en valores y competencias socioemocionales para docentes}

La mayoría de autores coinciden en que un docente debe tener, además deconocimiento didácticoyen el uso delas nuevas tecnologías, destrezas para comunicarse y relacionarse positivamente con los diversos agentes de la comunidad educativa y alumnado, capacidad para llevar a cabo procesos de autorregulación y hetero-regulación, competencia para liderar y manejar el aula, además de saber trabajar en equipo y capacidad de toma de decisiones responsable y ética para la resolución de problemas en el contexto educativo. Solamente en las propuestas más actuales, en consonancia con lo apuntado por López- 
Goñi y Zabala (2012), se introducen competencias intrapersonales, relacionadas por ejemplo con el autoconocimiento o estima personal, a pesar de que las propias emociones afectan la identidad profesional y las prácticas docentes (Buitrago-Bonilla \& CárdenasSoler, 2017). Finalmente, destaca la ética docente en cuanto a las responsabilidades que se espera que asuma el docente con la garantía del derecho de todas las personas a la educación, a una conciencia social y educativa crítica, al cuidado y a la personalización educativa, a la formación y al desarrollo del profesorado, a la implicación activa y a los compromisos con la comunidad educativa.

La necesidad de incorporar valores en la formación de las distintas etapas educativas, a la que suele denominarse con la expresión educar oformar en valores, es compartida por la mayoría de las instancias sociales (GUNI, 2008). En la universidad es un componente esencial de la formación integral de los estudiantes, para que estos se desarrollen como profesionales competentes y ciudadanos comprometidos con la mejora de la sociedad. Asimismo, los distintos ámbitos profesionales y sociales han asumido la presencia de los valores en sus organizaciones y en sus prácticas y han solicitado a los profesionales actuales y futuros un perfil que los incorpore.

Elestudiodecompetencias profesionales noes nuevo. Yaafinales del siglo XX algunos autores habían intentado delimitar y evaluar las competencias básicas de los docentes, relacionadas con el "buen hacer" en la profesión. Ahora bien, conocer el perfil profesional del docente actual, desde un modelo educativo descriptivo, implica analizar las competencias que debería desarrollar para responder a las demandas de la sociedad en nuestro contexto específico y en este momento histórico en concreto.

En esta línea, Gimeno-Sacristán (2010) propone que los cambios sociales y educativos requieren de nuevas competencias básicas o transversales docentes, que se clasifican así:

1. Nuevas exigencias en el proceso formativo:

- Énfasis del carácter práctico de los estudios de magisterio y la relación teoría-práctica.

- Acentuación de la formación del docente como profesional responsable y capacitado para tomar decisiones innovadoras, a través del trabajo en equipo en el centro educativo. 
- Capacidad de actuación buscando las sinergias de otros agentes sociales que pueden facilitar el éxito del trabajo desarrollado en la escuela: familias, asociaciones, entidades, autoridades educativas, entre otros.

2. Adquisición de conocimientos y acceso a la información:

- Capacitación para desarrollar su labor en la sociedad del conocimiento.

- Conocimiento de los nuevos procesos de formación, que las tecnologías de la información y la comunicación proponen.

3. Necesidad de formación personal y en habilidades sociales:

- Junto con la preparación técnica y profesional, se requiere una sólida formación personal. Aspectos como el autoconocimiento, la estima personal, la capacidad de establecer relaciones de grupo constructivas, la actitud solidaria y democrática, son esenciales.

- Habilidades sociales para ejercer el liderazgo, que es propio de la función docente, en los grupos de alumnos y alumnas que deberá conducir.

- Preparación para trabajar en equipo con el resto del profesorado.

Por otro lado, hace dos décadas surgió un creciente interés en el estudio de cómo aprender a ser y a vivir juntos (Delors, 1996) y en su influencia en el aprendizaje y la adaptación social. Diversos estudios en el campo del desarrollo evolutivo relacionan una alta habilidad en competencias emocionales y sociales [CES] con mayor bienestar, climas positivos de trabajo y rendimiento escolar o laboral, mientras que una baja habilidad en estas competencias puede conllevar dificultades en el ámbito personal, social, académico y profesional (Damon, Lerner \& Eisemberg, 2006). Por ejemplo, en un estudio reciente sobre satisfacción vital en adolescentes, se observa cómo la competencia de autoestima y regulación emocional son grandes predictores, junto al clima escolar positivo, de dicho bienestar, incluso cuando el joven ha sido víctima de bullying (Lázaro-Visa, Palomera, Briones, Fernández-Fuertes \& Fernández-Rouco, 2019).

Dichos beneficios se pueden conseguir si los docentes adquieren CES e implementan programas de aprendizaje socio-emocional [ASE]. Por ello los investigadores demandan la promoción del 
ASE, no solo en los niños sino también para los propios docentes (Greenberg et al., 2003; Mansfield, Beltman, Broadley \& WeatherbyFell, 2016). El Departamento de Educación y Habilidades del Reino Unido en su informe Every Teacher Matters (Bassett, Haldenby, Tanner \& Trewhitt, 2010) concluye con la recomendación del desarrollo específico de CES, tanto en las escuelas como en las instituciones para la formación docente, basándose en la idea de que una competencia que no se ha adquirido no se puede enseñar, como tampoco es posible la calidad en la enseñanza ante la ausencia de bienestar docente.

\section{Descripción de la asignatura}

\section{Objetivos}

La concepción de competencias básicas, en particular aquellas necesarias para un desempeño docente de calidad, con ética y con bienestar, es el eje vertebrador de la asignatura. En este sentido, la planificación y organización docente responden al planteamiento del EEES y del ASE, al entender que el rol a adoptar como docentes servirá como mediador entre la realidad social y el alumnado (Moreira, 2000). Los objetivos se dirigen a:

1. Reflexionar sobre la forma de ser y estar propia y del otro.

2. Aprender y poner en práctica estrategias para el propio desarrollo socioemocional y ético.

3. Promover el bienestar docente y la convivencia en la comunidad educativa.

Los resultados esperados de aprendizaje, por su parte, se concretan en:

1. Mostrar conocimientos acerca de los fundamentos teóricos de las competencias socioemocionales y valores universales desde el marco de la psicología positiva.

2. Capacidad de trabajar en equipo de forma empática, responsable, cooperativa y creativa.

3. Adecuada capacidad de argumentación, exposición de ideas y escucha activa.

4. Consciencia de los valores y fortalezas personales 


\section{Ejes temáticos}

El diseño curricular propuesto a continuación se enmarca en el campo de la psicología positiva, en relación con los rasgos de resiliencia docente y fortalezas humanas; igualmente, en consonancia con las demandas de competencias docentes analizadas desde el análisis de tareas docentes. La intervención se focaliza en cinco CES, que corresponden con el modelo propuesto por la Collaborative for Academic, Social, and Emotional Learning (2003) para el ASE, por ser el modelo más ampliamente utilizado a nivel internacional, estar enfocado al contexto educativo y por ser integrador tanto de competencias emocionales y sociales como del desarrollo moral de la persona. Los siguientes ejes temáticos corresponden a las CES que nos proponemos desarrollar, de tal manera que los estudiantes aprendan su conceptualización y realicen prácticas experienciales dirigidas a su propio desarrollo personal.

\section{Formación personal del docente.}

Consiste en la explicación de la fundamentación teórica de la educación en valores y CES en la formación del profesorado. Además, a través del método expositivo y técnicas dialógicas se abordan los diferentes enfoques epistemológicos de la asignatura, así como el acercamiento a la metodología propia del ASE y las razones científicas, académicas y profesionales por las que los docentes necesitan desarrollar CES. Con esta introducción finalmente se promueven conocimientos y actitudes positivas hacia el desarrollo integral en la educación y, en particular, en la formación docente para incrementar la calidad educativa, prevenir dificultades profesionales y ejercer un liderazgo auténtico.

\section{Toma responsable de decisiones.}

Se trabaja la toma de decisiones basadas en el examen de las normas éticas y consecuencias probables de diferentes acciones. Se parte del autoconocimiento de los propios valores personales, así como la aplicación de las habilidades de toma de decisiones a situaciones académicas y sociales. Para ello, se analiza la conceptualización de valores, desarrollo moral y aprendizaje ético, así como los valores universales a través de la Convención de Derechos Humanos y Derechos de la Infancia. 


\section{Conciencia de uno mismo.}

Se aborda teóricamente y se ofrecen espacios para la reflexión sobre el autoconcepto, el propio reconocimiento y estima. Se trabaja la evaluación con precisión de los propios sentimientos, intereses y fortalezas, y el mantenimiento de un sentido bien fundamentado de confianza en uno mismo. Para ello, se manejan junto con los estudiantes fuentes y estrategias relativas a la conceptualización del autoconcepto y sus dimensiones, y a una autoestima sana y óptima. También se reflexiona sobre la influencia de estas competencias sobre diversos ámbitos vitales.

\section{Autorregulación.}

Trabajamos la regulación de las emociones de uno mismo para manejar el estrés, controlar la impulsividad y perseverar en la superación de obstáculos, así como en la promoción de bienestar; para establecer y monitorear el logro de metas personales y académicas; y para expresar emociones de manera apropiada. Para ello, comenzamos aproximándonos al concepto de emoción y, a través del modelo de IE de Mayer y Salovey (1997), avanzamos hacia la comprensión de los procesos previos de identificación y comprensión emocional que requiere la regulación emocional tanto intra- como interpersonal. Después, profundizamos en el conocimiento de la regulación emocional a través de su delimitación conceptual, funcionamiento e influencia sobre el ajuste psicosocial. Finalmente se ponen en práctica diversas estrategias propias de una regulación emocional efectiva y adaptativa.

\section{Conciencia social.}

Se aborda la conceptualización de la empatía, sus componentes, su importancia en la vida y en el rol docente y los estilos empáticos en la docencia. Así mismo, se practican estrategias para promover la capacidad de ponerse en el lugar de los demás y empatizar a nivel emocional, cognitivo y conductual - escucha activa, comunicación no verbal-.

\section{Habilidades de relación.}

Se estudian teóricamente y mediante la reflexión y entrenamiento las habilidades sociales para establecer y mantener relaciones sanas y positivas basadas en la cooperación; comprender 
los derechos asertivos y estilos de comunicación; resistir la presión social inapropiada; prevenir, gestionar y resolver creativamente conflictos interpersonales; y buscar ayuda cuando sea necesario.

\section{Metodología}

A lo largo del desarrollo de las clases, hemos implementado diversas metodologías en función del objetivo: aprendizaje basado en problemas [ABP] (Hmelo-Silver, 2004), estudios de caso, aprendizaje dialógico - presencial y por internet-, aprendizaje experiencial, aprendizaje cooperativo -técnica puzzle- (Aronson \& Patnoe, 2011), simulaciones, teatro-foro (Motos-Teruel \& Navarro-Amorós, 2012), portafolio o cuaderno de bitácora; todo ello apoyado en recursos documentales y audiovisuales a través de la plataforma virtual Moodle.

También seguimos las recomendaciones de Kornacki y Caruso (2007), quienes establecen una serie de orientaciones para la educación emocional en población adulta, tales como: insertar dinámicas de cohesión grupal; utilizar metodologías vivenciales y de experimentación, donde se utilizan los recursos y experiencias personales de los asistentes; trabajar con métodos grupales y cooperativos; hacer retroalimentación y evaluaciones que informen de resultados; y dejar tiempos para la reflexión.

Seguir una metodología vivencial y activa supone disponer de tiempos flexibles y un espacio abierto para las dinámicas grupales, de modo que a nivel organizativo solicitamos a la Facultad de Educación la distribución de las cuatro horas de la asignatura, agrupadas en dos sesiones de dos horas seguidas, y la facilitación en lo posible del aula de música - sillas de pala y espacio libre$\mathrm{u}$ otros espacios diáfanos de la universidad - salas del pabellón polideportivo-. En alguna ocasión también hemos utilizado los espacios de encuentro social UC o el espacio público.

Acontinuación, ofrecemosunasíntesisdelasfasesmetodológicas en las que se pueden simplificar los avances realizados a través de la investigación-acción durante los diez años de docencia de la asignatura FVCPD con el objeto de facilitar la visión longitudinal del proyecto. En todo este proceso, hemos diseñado metodologías de intervención y evaluación, así como también, de forma paralela, diversas investigaciones para observar los resultados 
e ir perfeccionando el proyecto a través de la experiencia. Todo ello ha sido difundido a lo largo de los años a través de reuniones científicas, publicaciones y formaciones externas.

\section{Primera fase: desde su creación hasta el primer Proyecto de Innovación Docente [PID] basado en el ABP avalado por la UC - 2010-12-.}

Llevamos a cabo ABP mediante técnica puzzle para instruir en Derechos Humanos y de la Infancia, y su defensa y promoción en los contextos educativos.

Además, los estudiantes preparan exposiciones sobre los contenidos de la asignatura con sus grupos de "expertos" repartidos por cada tema del programa-. Estas exposiciones son grabadas para retroalimentarlos en tutorías mediante su visualización para la mejora de competencias comunicativas.

La técnica de ABP también se usa mediante co-docencia y creación de grupos de estudiantes inter-clases para facilitar la integración y desarrollo de las competencias comunes de dos asignaturas del área de Psicología Evolutiva y de la Educación. Se analizan y resuelven 12 situaciones reales o cuasirreales habituales de la labor docente diseñadas a tal efecto - por ejemplo: regulación emocional y burnout, relaciones profesionales, gestión del aula, e innovación-.

La evaluación de la asignatura es continua y participativa. Así los estudiantes se involucran en la evaluación de las competencias propias y de los compañeros. Para ello se emplean dos rúbricas. En una de ellas se evalúa el proceso - la actitud y el compromiso en las actividades de grupo- propio - autoinforme- y de cada integrante -heteroinforme-. Esas evaluaciones suponen el 10\% de la evaluación. Para evaluar el producto, es decir las exposiciones y trabajos de los grupos de expertos, se emplea una rúbrica utilizada por el profesor y otros dos grupos de expertos que actúan de observadores-evaluadores en el aula -15\%-. La participación de los estudiantes es considerada, pues supone el 3.5\% de la calificación. Además, la evaluación de las competencias socioemocionales cuenta con una prueba objetiva final sobre los contenidos teóricos $-30 \%-$. Finalmente realizan el análisis y resolución de un caso de forma grupal en relación con competencias socioemocionales $-15 \%-$ y valores $-26.5 \%-$. 


\section{Segunda fase: optimización de la metodología dialógica -segundo PID en la UC, 2012-15-.}

Llevamos a cabo una metodología basada en el diálogo y el debate para favorecer la clarificación de valores, propia y en situaciones de conflicto, y trabajar la argumentación dialógica en el posicionamiento personal.

Para favorecer la escucha y reconocimiento de diversos posicionamientos, el pensamiento crítico y la toma de perspectiva, se fortaleció la metodología dialógica al incorporar el debate en línea entre grupos heterogéneos procedentes de distintas universidades, culturas y generaciones.

Con el objetivo de promover el interés de los estudiantes en el desarrollo de competencias personales, se propuso:

- $\quad$ En grupo de expertos: la exposición, diseño y puesta en marcha de actividades en el aula, y la elaboración de preguntas tipo test sobre cada tema. La composición de un tuit creativo a modo de titular o moraleja de cada tema que recibían seguidores de su grupo-aula.

- Por grupos conformados al azar: la elaboración de un proyecto de investigación con el objeto de construir el perfil con los valores y competencias personales que se suelen asociar a un maestro auténtico.

- Voz del alumnado: el último tema del programa es propuesto por intereses y votación en línea del alumnado de cada titulación.

La metodología activa y vivencial requirió de un espacio diáfano y estimulante. Espacios deportivos, sociales y públicos de la UC comenzaron a ser empleados para desarrollar este proyecto. Además, se abre la clase al contexto mediante la invitación de profesionales vinculados con la oferta en materia de voluntariado y la liga de debates ofertados por la UC.

Se continúa con la misma metodología de evaluación. 


\section{Tercera fase: fortalecimiento de la sensibilidad y reflexión sociomoral, y comprensión crítica a través del aprendizaje dialógico y el ABP transversal -2015-18-.}

La formación dialógica se dirige, además de a objetivos presentes en fases anteriores, a potenciar la sensibilidad moral, la reflexión sociomoral y la comprensión crítica de la realidad mediante la actividad de los reporteros de dilemas reales -en internet y grupal-. En grupos crean un dilema moral real, que publican en la plataforma Moodle - una vez revisado por la profesora-. El más votado es el segundo dilema a debatir (Briones, Palomera \& Gómez-Linares, 2018).

El desarrollo socioemocional, creativo y ético se potencia mediante un enfoque ABP transversal. Se analiza un caso que refleja conflictos propios de la vida académica. En grupos de expertos, analizan la competencia de la que son responsables, proponen estrategias para su optimización y desarrollan una, gestionando una actividad en el aula.

Además, en grupo de expertos plantean y resuelven una pregunta "curiosa" para profundizar en su temática.

Se optimiza la evaluación formativa y sumativa:

- $\quad \mathrm{Al}$ ofrecer ejemplos de resolución de caso y dilema.

- Al dar retroalimentación de participación, resultados y orientaciones de mejora.

- Con la optimización de los instrumentos de evaluación - rúbricas con el grado de presencia de cada criterio de evaluación- y las técnicas de evaluación - autoinformes de la propia actuación y de la experiencia-.

\section{Cuarta fase: integración de metodologías: bitácora, diálogo, ABP y teatro foro - 2018-19-}

La formación en valores continúa la metodología dialógica, con el diseño de un dilema moral habitual en estudiantes de magisterio.

Laformación competencial personal se refuerza con la propuesta de un cuaderno de bitácora personal para favorecer el metaconocimiento y la transferencia de lo aprendido a sus vidas. Responden a dos preguntas: ¿qué he aprendido? y ċcómo aplicarlo en mi día a día? 
La metodología de ABP se emplea para analizar un caso basado en una situación vivida por un estudiante de magisterio, que deben explicar y solucionar aplicando conocimientos.

Introducimos la metodología de teatro del oprimido para favorecer la asimilación de las competencias personales. A partir de un $\mathrm{ABP}$ donde los grupos identifican problemas docentes, elaboran un guion para su representación teatral, donde los resuelven aplicando lo aprendido. En las sesiones con teatro foro se analiza y se mejora cada problema grupal entre todos. Finalmente, los grupos graban su versión final en formato de corto cinematográfico, los cuales son vistos y premiados por los equipos en clase.

Se elimina el examen de contenidos teóricos y se evalúa el cuaderno de bitácora individual $-20 \%-$, la resolución del dilema moral $-25 \%-$ , el análisis y resolución de un caso $-25 \%$ - y la identificación, análisis y representación de un problema docente $-30 \%-$.

\section{Resultados}

Los principales resultados alcanzados, así como la valoración de la experiencia, han sido publicados (Briones \& Gómez-Linares, 2016; Briones \& Lara, 2016; Palomera, Briones \& GómezLinares, 2017; Palomera, Briones, Gómez-Linares \& Vera, 2017) y presentados en diversos foros científicos (Briones, Gómez-Linares \& Palomera, 2014, 2015a, 2015b; Briones et al. 2018; GarcíaRodicio et al., 2012; Gómez-Linares, Briones, Palomera \& Gómez, 2012; Palomera, 2013, 2014, 2016; Palomera, Briones \& GómezLinares, 2016).

De la valoración realizada en relación con el método $\mathrm{ABP}$ destaca la elevada satisfacción de los estudiantes con la experiencia educativa, así como su implicación en la resolución del caso propuesto (Briones \& Gómez-Linares, 2016). Esta experiencia y los casos analizados les parecen interesantes y de utilidad para su formación como docentes. Por otra parte, los resultados muestran que los estudiantes resuelven con éxito la actividad. En concreto, el 86\% de los equipos de alumnos elaboró informes de calidad, con notas correspondientes a las calificaciones de notable y sobresaliente. Por tanto, parece que se logró despertar en alguna medida la motivación intrínseca de los estudiantes y un aprendizaje 
profundo de los contenidos de las materias al permitir el uso de sus conocimientos de forma productiva, tal como se obtuvo en estudios previos (Walker \& Leary, 2009). En términos generales la percepción de los profesores responsables de la experiencia de innovación fue también positiva, como viene siendo habitual entre los docentes universitarios implicados en la práctica de metodologías activas (Arregi, Bilbatua \& Sagasta, 2004). Las principales dificultades y propuestas de mejora analizadas por el profesorado mediante un DAFO se relacionan con la coordinación y organización de la experiencia, así como con el reto de manejar el alto grado de diversidad de los perfiles docentes, los grupos de estudiantes y los contenidos de los casos.

Por otro lado, los resultados obtenidos mediante la metodología dialógica y el uso de dilemas morales demostraron la extraordinaria acogida de la técnica por parte de los estudiantes, tanto por sus valoraciones sobre la misma como por las consecuencias que identificaban había tenido esta en sus vidas. Por otra parte, obtuvimos evidencia sobre el potencial de la propuesta educativa para promover competencias discursivas y argumentativas y toma de perspectiva. No obstante, también se detectó una cierta dificultad para realizar reflexiones en las cuales se analice en profundidad distintas perspectivas, lo cual nos llevó a pensar en la necesidad de garantizar debates en los que se ofrezcan distintos puntos de vista, estilos argumentativos y ejemplos experienciales; que muevan a la reflexión y que contribuyan al esfuerzo por expresarse y respetar distintos planteamientos.

A continuación, ofrecemos ejemplos de categorización de las respuestas de los estudiantes a las cuestiones: ¿crees que estos ejercicios de dilemas te han ayudado en algo en tu vida diaria? ¿En qué principalmente? (Briones et al., 2014):

1. Permiteelautoconocimiento:mepermitióconocermis “valores", "conocimientos", "reacción ante distintas situaciones".

2. Potencia la capacidad reflexiva: "pensar sobre las situaciones", "considerar las consecuencias, motivaciones", "obtener conclusiones de mis actos".

3. Facilita la toma de perspectiva: "considerar otros puntos de vista", "ponerme en diferentes situaciones", "comprender otras ideas". 
4. Aporta conocimientos del mundo: "darme cuenta de lo que sucede a mi alrededor"

5. Incita a un cambio actitudinal: "tener más paciencia", "ser más tolerante", "tener en consideración al otro".

6. Conocimientos aplicados al aulay a la futura profesión: "contribuir mejor en los debates del aula.", "ver qué tipo de maestra quiero llegar a ser".

Con la incorporación del debate en línea con estudiantes de otras universidades internacionales -UAChile, UAMéxico, Grand Valley University - y nacionales - Universidad de la Experiencia en UPV-EHU-, con el objetivo de enriquecer los puntos de otras culturas y generaciones, los estudiantes presentaron nuevamente efectos positivos en habilidades propias del pensamiento crítico. Además, apreciaron el debate con personas con ideas distintas y el ejercicio de comprensión, razonamiento y reflexión que la actividad conlleva (Briones et al. 2015b; Briones \& Lara, 2016).

En cuanto al aprendizaje de CES, en un estudio de 2012 (Palomera, Briones, Gómez-Linares \& Vera, 2017) exploramos el impacto del programa de formación. Llevamos a cabo una investigación cuasiexperimental con medida pretest y postest y grupo control - de otras facultades de educación de UVA y UPV-EHU-. Los estudiantes respondieron de forma anónima cuestionarios validados que evaluaban algunas de las competencias entrenadas. También se evaluó la deseabilidad social y los cinco grandes factores de la personalidad, como medidas de control. Los resultados demostraron que el programa había tenido un impacto significativo en la autoestima, la empatía, la confianza como orador y el afecto negativo de los estudiantes. Estos resultados son de gran valor científico, dada la ausencia histórica de formación en este ámbito a nivel formal y de otras experiencias comparables.

Además, mediante un estudio cualitativo de 2013 (Briones, Palomera \& Gómez-Linares, 2014) con una encuesta semiestructurada, anónima y por escrito, comprobamos la valoración de la experiencia educativa por parte de los estudiantes. El análisis y categorización de la información aportada sobre cuatro preguntas abiertas - sobre su aprendizaje, lo que les ha gustado más y menos, y sugerencias para la mejora de la asignatura-, nos permiten documentar que el alumnado percibe haber aprendido valores y 
competencias para la vida personal y profesional, como conocer y valorarse a uno mismo y a los demás, afrontamiento positivo y autorregulación, habilidades sociales y resolución de dilemas, y a ser más feliz. Además, detectamos que a los estudiantes les había gustado la metodología participativa, dialógica y experiencial vivida. En concreto, realizaron comentarios positivos relativos a la exposición y prácticas realizadas en grupos, la relajación y el clima de libertad creativa, confianza y diversión creado en el aula.

En estudios posteriores (Briones et al. 2018), sin embargo, se obtiene que, si bien los estudiantes perciben haber alcanzado diversos aprendizajes gracias a esta formación - autoconocimiento, comprensión de valores y dilemas, empatía y perspectiva social, comprensión y autorregulación-, no se obtienen efectos significativos en el razonamiento moral de los estudiantes, medido antes y después de la formación mediante una versión del cuestionario de problemas sociomorales (adaptación española del DIT, Pérez-Delgado, Mestre, Frías \& Soler, 1996). De tal manera, se comprueba que los altos porcentajes en los estadios propios de una moral convencional $-76.4 \%$, frente al $16.1 \%$ de estudiantes en una moral posconvencional- se mantienen después de la formación. Tampoco debe sorprender este resultado, si se contempla que se trata de estudiantes de primer curso, y que incluso se ha evidenciado que estudiantes universitarios de los últimos cursos académicos suelen situarse en el nivel convencional de la escala de Kohlberg (Guerrero \& Gómez, 2013). Estos hallazgos nos llevan a plantear que, para próximas ediciones, será necesario atender primero a su motivación para involucrarse en diversas actividades en clase y en internet, que favorezca su formación para la argumentación y la reflexión crítica. Además, como conclusión se subraya la necesidad de una mayor investigación sobre herramientas para la evaluación y la formación ética y moral en la educación superior.

Debido a que la creatividad es una competencia indirectamente estimulada a través de las exposiciones - con el diseño de actividades innovadoras, formato de exposición creativo y formulación de preguntas nuevas-, quisimos observar los efectos sobre ella. En el curso 2015 realizamos una evaluación cuasiexperimental. Los resultados muestran mejoras significativas en su concepción sobre la creatividad -innata o posible de mejorar-, su autoconcepto y autoeficacia creativa (Palomera, Briones \& Gómez-Linares, 2016). 
Finalmente, en el presente curso académico, con la introducción de la técnica de teatro foro, los estudiantes han mostrado una alta motivación y satisfacción con la experiencia, en la que han tenido que superar su miedo escénico. Han valorado especialmente el apoyo y trabajo en equipo. Actualmente los análisis llevados a cabo muestran una mejora significativa en los niveles de empatía de los estudiantes, al comparar las puntuaciones pre y postest.

La conclusión más importante de los estudios es que es posible mejorar las competencias socioemocionales, la clarificación de valores y razonamiento moral en la formación inicial de los maestros, lo cual nos estimula a seguir desarrollando el modelo educativo e introducir algunas mejoras metodológicas con objeto de generar un mayor impacto en las competencias menos desarrolladas, así como de estudiar empíricamente el efecto en otras en las que no se ha probado aún su eficacia.

\section{Posibilidades de transferencia a otros ámbitos o áreas de la educación superior}

En relación con la transferencia de la experiencia a otros ámbitos más allá de la innovación docente en las titulaciones de grado en magisterio, algunas de ellas ya se han realizado a lo largo de estos años, y otras las consideramos factibles, como comentaremos a continuación.

Por un lado, en el ámbito académico, se impulsó la continuidad de la formación ofertada en este proyecto, a través de un Máster Título Propio en Educación Emocional, Social y de la Creatividad en la UC, en convenio con la Fundación Botín, el cual comenzó en el año 2011.

De la misma manera, a través de la formación Sénior en la UC estamos llevando a cabo el curso monográfico titulado Desarrollo psicológico y bienestar en la edad adulta. Desde este curso se ofrece formación en el marco conceptual del desarrollo y bienestar psicológico en la edad adulta, donde se atiende a las tareas y desafíos propios de la vida adulta y se entrenan competencias personales -autoconcepto, autoestima y autorregulación emocional- e interpersonales -empatía, asertividad y resolución de conflictos interpersonales-, con el objetivo de alcanzar un mayor desarrollo personal y de lograr una conexión social satisfactoria. 
Por otro lado, puesto que la formación transversal en valores y competencias personales de la UC es básica y obligatoria para todos los grados ofertados, consideramos que las metodologías creadas y validadas en estos años se podrían aplicar perfectamente en todos ellos con la variación de los ejemplos profesionales a utilizar y el empleo de los mismos recursos didácticos aquí explicados.

En el ámbito de la transferencia social, a lo largo de estos años hemos participado en múltiples formaciones dirigidas al desarrollo de las competencias personales en diversos ámbitos: formación de docentes en activo; formación de familias, a través de los centros educativos; formación de la tercera edad; y formación de profesionales de las artes escénicas. También hemos trabajado con transferencia a la inversa, mediante charlas y talleres a los estudiantes en colaboración con asociaciones sociales relacionadas con la salud mental, diversidad cultural, educación ambiental, contextos educativos formales y trabajo con población de riesgo de exclusión social. También se ha colaborado en el Programa de voluntariado de la UC y en el desarrollo de competencias transversales, con la identificación de parámetros del aprendizaje servicio y del voluntariado transformador, y su integración en una formación y evaluación que garantizan la efectividad de esta modalidad formativa de la educación superior (Briones, Rubio, Salcines, Escofet \& González, 2017; Cacho, Briones \& Llano, 2017).

Finalmente, también vemos transferible el proyecto en la formación de profesionales en activo de casi cualquier campo laboral, pues los valores y competencias personales son habilidades transversales cognitivas, del ámbito socioafectivo, presentes en cualquier ser humano y por tanto en cualquier ámbito de trabajo. 


\section{Referencias}

Aronson, E., \& Patnoe, S. (2011). Cooperation in the classroom: The jigsaw method (3rd ed.). London: Pinter y Martin, Ltd.

Arregi, X., Bilbatua, M., \& Sagasta, M. (2004). Innovación curricular en la Facultad de Humanidades y Ciencias de la Educación de Mondragón Unibertsitatea: Diseño e implementación del perfil profesional del Maestro de Educación Infantil. Revista Interuniversitaria de Formación del Profesorado, 18(1), 109-129.

Bassett, D., Haldenby, A., Tanner, W., \& Trewhitt, K. (2010). Every teacher matters. London: Reform.

Briones, E., \& Gómez-Linares, A. (2016). Psicología para Docentes: Guía y casos resueltos aplicando el Aprendizaje Basado en Problemas (ABP). Madrid: Pirámide.

Briones, E., \& Lara, L. (2016). Educación ética en la Universidad a través del diálogo multicultural online. Comunicar, 47(XXIV), 99-107. https:// doi.org/10.3916/c47-2016-10

Briones, E., Gómez-Linares, A., \& Palomera, R. (2014). Universidad y valores personales: una apuesta por la internacionalización, la diversidad y el diálogo en la formación. En A. Calvo, C. Rodríguez-Hoyos, \& I. Haya, Libro de Actas del XIII Congreso Internacional de Formación del Profesorado (AUFOP) (pp. 1263-1272). Santander: AUFOPUniversidad de Cantabria.

Briones, E., Gómez-Linares, A., \& Palomera, R. (2015a). La formación en valores en el EEES: propuesta de instrumento, medio y técnicas de evaluación. En N. González, I. Salcines, \& E. García (Coords), Tendencias emergentes en evaluación formativa y compartida en docencia. El papel de las nuevas tecnologías (pp. 215-233). Santander: Editorial Universidad de Cantabria.

Briones, E., Gómez-Linares, A., \& Palomera, R. (2015b). Teaching in values in higher education: innovation by online dialogue between students from different universities. En M. Carmo (Ed.), Proceedings of the International Conference on Education and New Developments 2015 (pp. 194-198). Lisboa: WIARS.

Briones, E., Palomera, R., \& Gómez-Linares, A. (2014). Dilemas de la formación en valores en los grados de Magisterio. Ponencia presentada en el XI Foro Internacional sobre la Evaluación de la Calidad de la Investigación y de la Educación Superior (FECIES). Bilbao (España).

Briones, E., Palomera, R., \& Gómez-Linares, A. (2018). Programa para la formación en valores de futuros docentes. Comunicación presentada 
en el IX Congreso Internacional de Psicología y Educación. Logroño (España).

Briones, E., Rubio, L., Salcines, I., Escofet, A., \& González, N. (2017). Instrumentos cualitativos para la evaluación del Programa de voluntariado desde el APS. 5th Internacional Congress of Educational Sciences and Development. Santander.

Buitrago-Bonilla, R., \& Cárdenas-Soler, R. (2017). Emociones e identidad profesional docente: relaciones e incidencia. Praxis \& Saber, 8(17), 225-247. doi.org/10.19053/22160159.v8.n17.2018.7208

Cacho, Y., Briones, E., \& Llano, L. (2017). Voluntariado universitario $y$ aprendizaje de competencias. 5th Internacional Congress of Educational Sciences and Development. Santander.

Collaborative for Academic, Social, and Emotional Learning. (2003). Safe and sound: An educational leader's guide to evidence-based social and emotional learning (SEL) programs. Chicago: Author.

Damon, W., Lerner, R., \& Eisenberg, N. (Eds.). (2006). Handbook of Child Psychology: Volume Three: Social, Emotional, and Personality Development. New York: Wileyy Sons.

Delors, J. (1996). La educación encierra un tesoro. Madrid: UnescoSantillana.

García-Rodicio, H., Gómez-Linares, A., Briones, E., Palomera, R., Fernández-Fuertes, A., Gómez-Pérez, E... Hernández, A. (2012). Research y Resolve Project (RyR): An example of an innovative curriculum design to promote professional competences in preservice teachers. INTED2012. Valencia

Gimeno-Sacristán, J. (2010). La carrera profesional para el profesorado. Revista Interuniversitaria de Formación de Profesorado, 68, 24326o. Recuperado de http://aufop.com/aufop/uploaded_files/ articulos/127926880o.pdf

Gómez-Linares, A., Briones, E., Palomera, R., \& Gómez, E. (2012). Formación en Valores y Competencias Personales para Docentes a través del Aprendizaje Activo. Actas del V Congreso Internacional de Estilos de Aprendizaje (pp. 1-10), Santander.

Greenberg, M., Weissberg, R., O’Brien, M.., Zins, J., Fredericks, L., Resnik, H., \& Elias, M. (2003). Enhancing school-based prevention and youth development through coordinated social, emotional, and academic learning. American Psychologist, 58(6-7), 466-474.

Guerrero, M., \& Gómez, D. (2013). Enseñanza de la ética y la educación moral, ¿permanecen ausentes de los programas universitarios?. 
Revista Electrónica de Investigación Educativa, 15(1), 122135. Recuperado de https://dialnet.unirioja.es/descarga/ articulo/4397659.pdf

GUNI. (2008). La Educación Superior en el Mundo 3. Madrid: MundiPrensa.

Hmelo-Silver, C. (2004). Problem-Based Learning: what and how do students learn? Educational Psychology Review, 16(3), 235-266. https://doi.org/10.1023/B:EDPR.oooo034022.16470.f3

Kornacki, S., \& Caruso, D. (2007). A theory-based, practical approach to emotional intelligence training: ten ways to increase emotional skills. En J. Ciarrochi \& J. Mayer (Eds.), Applying Emotional Intelligence: A Practitioner's Guide (pp. 53-88). New York: Psychology Press.

Lázaro-Visa, S., Palomera, R., Briones, E., Fernández-Fuertes, A., \& Fernández-Rouco, N. (2019). Bullied Adolescent's Life Satisfaction: Personal Competencies and School Climate as Protective Factors. Frontiers in Psychology, 10. https://doi.org/10.3389/ fpsyg.2019.01691

López-Goñi, I., \& Zabala, J. (2012). La competencia emocional en los currículos de formación inicial de los docentes. Un estudio comparativo. Madrid: Ministerio de Educación.

Mansfield, C., Beltman, S., Broadley, T., \& Weatherby-Fell, N. (2016). Building resilience in teacher education: An evidenced informed framework. Teaching and Teacher Education, 54, 77-87. https://doi. org/10.1016/j.tate.2015.11.016

Mayer, J., \& Salovey, P. (1997). What is emotional intelligence?. En P. Salovey \& D. Sluyter (Eds). Emotional Development and Emotional Intelligence: Implications for Educators (pp. 3-31). New York: Basic Books.

Moreira, M. (2000). Aprendizaje significativo: teoría y práctica. Madrid: Visor.

Motos-Teruel, T., \& Navarro-Amorós, A. (2012). Estrategias del Teatro del Oprimido para la formación permanente del profesorado. Magis, Revista Internacional de Investigación en Educación, 4(9), 619-635. Recuperado de https://revistas.javeriana.edu.co/index.php/MAGIS/ article/view/3579/2693

Palomera, R. (2013). La asignatura pendiente: La formación en fortalezas personales del docente del Siglo XXI. Conferencia plenaria en las Jornadas de Psicología Positiva. Claves Psicológicas para una vida más plena. Madrid. 
Palomera, R. (2014). En busca de las competencias perdidas del docente del siglo XXI. X Congreso Internacional de la SEAS, Valencia.

Palomera, R. (2016). Desmontando la casa por el tejado: Innovaciones educativas en la formación superior de docentes. Conferencia plenaria, III Congreso Nacional de Psicología Positiva, Baeza, Jaen.

Palomera, R., Briones, E., \& Gómez-Linares, A. (2016). Innovar en educación activando la creatividad de los futuros docentes. VIII Congreso Internacional de Psicología y Educación (CIPE 2016). Alicante.

Palomera, R., Briones, E., \& Gómez-Linares, A. (2017). Diseño, desarrollo y resultados de un programa de Educación Socio-Emocional para la formación de docentes a nivel de Grado y Postgrado. Contextos Educativos. Revista de Educación, (20), 165-182. https://doi. org/10.18172/con.2988

Palomera, R., Briones, E., Gómez-Linares, A., \& Vera. J. (2017). Filling the gap: improving the social and emotional skills of pre-service teachers. Revista de Psicodidáctica, 22(2), 142-149. https://doi.org/10.1016/j. psicod.2017.05.002

Pérez-Delgado, E., Mestre, V., Frías, M., \& Soler, M. (1996). El cuestionario de problemas sociomorales (DIT) de J. Rest (Manual). Valencia: Nau Llibres.

Walker, A., \& Leary, H. (2009). A Problem Based Learning Meta Analysis: Differences Across Problem Types, Implementation Types, Disciplines, and Assessment Levels. Interdisciplinary Journal of Problem-based Learning, 3, 12-43. https://doi.org/10.7771/15415015.1061 